\title{
Thermo-Chemo-Electro-Mechanical Formulation of Saturated Charged Porous Solids
}

\author{
J. M. HUYGHE and J. D. JANSSEN \\ Department of Mechanical Engineering, Eindhoven University of Technology, Eindhoven, \\ The Netherlands
}

(Received: 4 July 1997; in final form: 10 December 1997)

\begin{abstract}
A thermo-chemo-electro-mechanical formulation of quasi-static finite deformation of swelling incompressible porous media is derived from a mixture theory including the volume fraction concept. The model consists of an electrically charged porous solid saturated with an ionic solution. Incompressible deformation is assumed. The mixture as a whole is assumed locally electroneutral. Different constituents following different kinematic paths are defined: solid, fluid, anions, cations and neutral solutes. Balance laws are derived for each constituent and for the mixture as a whole. A Lagrangian form of the second law of thermodynamics for incompressible porous media is used to derive the constitutive restrictions of the medium. The material properties are shown to be contained in one strain energy function and a matrix of frictional tensors. A principle of reversibility results from the constitutive restrictions. Existing theories of swelling media should be evaluated with respect to this principle.
\end{abstract}

Key words: mixture theory, biology, hydrogel, shale, polymer.

\section{Nomenclature}

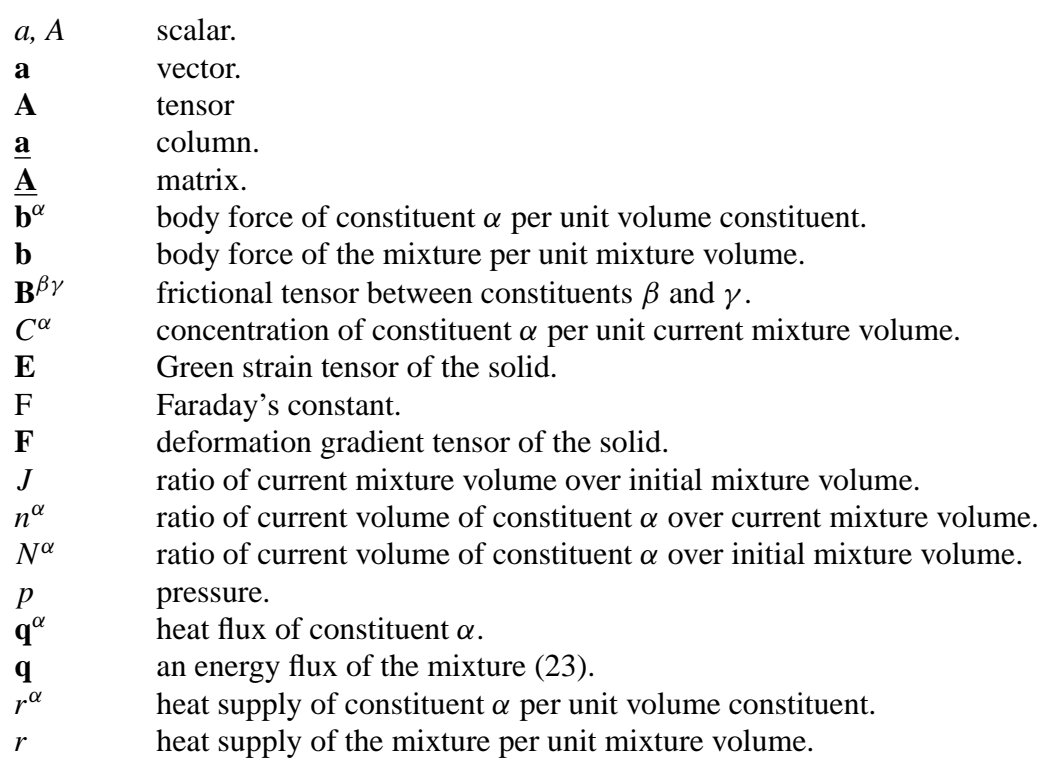




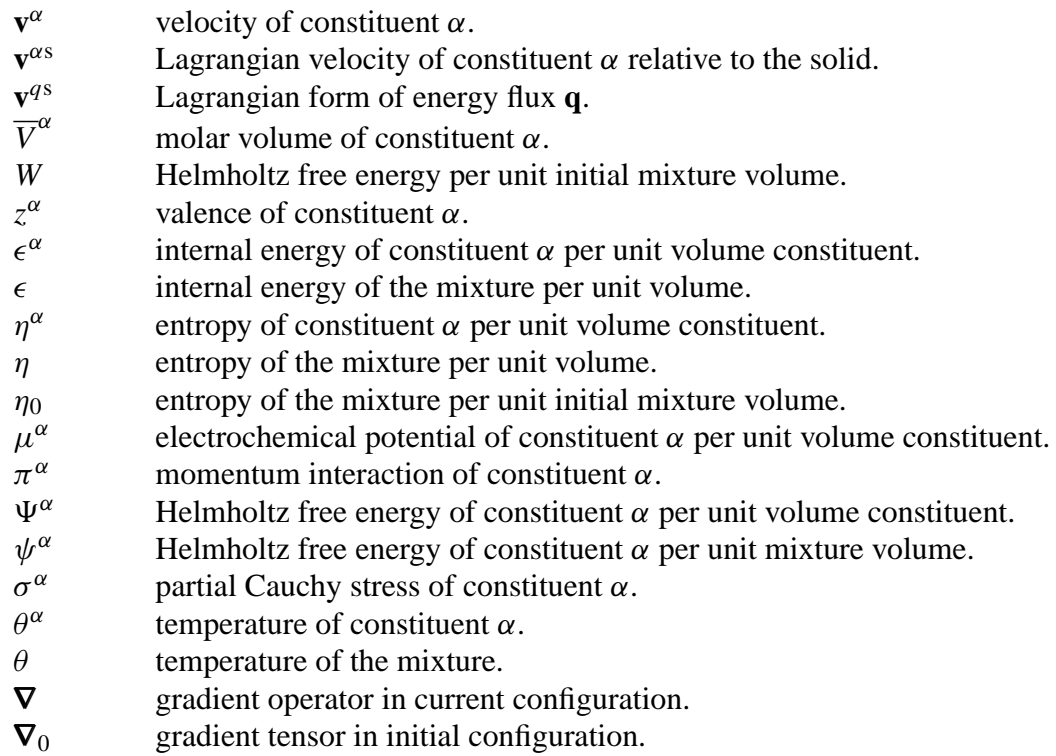

\section{Introduction}

Many biological, mineral and synthetic porous media exhibit swelling or shrinking when in contact with changing salt concentrations. This phenomenon, observed in clays, shales, cartilage and gels, is caused by electric charges fixed to the solid, counteracted by corresponding charges in the fluid. These charges result in a variety of features, including swelling, electro-osmosis, streaming potentials and streaming currents. In physiology, quantitative understanding of both intracellular and extracellular events requires correct modelling of these phenomena in deforming tissue. At least four components are involved in the swelling mechanics: a solid; a fluid; anions; and cations. Lai et al. [7] have realized a major breakthrough by developing a triphasic theory for soft hydrated tissue. The three phases involved are the solid, the fluid and the ionic phase. They have applied the theory to cartilage while neglecting geometric nonlinearities. They have verified the theory for one-dimensional equilibrium results of swelling. Sherwood [8], working in the field of petroleum engineering, has presented a triphasic model of shale poroelasticity which, unlike the theory of Lai et al. does not include electrical charges. Snijders et al. [10] have developed a family of finite elements for two-dimensional, three-dimensional and axisymmetric analysis of finite deformation of triphasic media. This novel finite element software features fixed charge density as one of its inputs. However, they use it only to model Donnan osmotic swelling and ignore both electrical current and electric potential fields within the medium. Oomens et al. [14] have performed swelling pressure experiments and confined swelling and compression experiments on an acrylamide acrylic acid copolymer gel and have found the gel properties analoguous to properties of soft charged hydrated tissue as measured by Drost et al. [13]. The model of Snijders 
Table I. Direct and coupled flow phenomena in charged porous media

\begin{tabular}{lllll}
\hline Flow/force & Pressure & Chemical potential & Electric potential & Temperature \\
\hline Fluid & filtration & osmosis & electro-osmosis & thermo-osmosis \\
Solute & ultrafiltration & diffusion & electroforesis & thermal diffusion \\
Current & streaming current & diffusion current & electric conduction & thermo-electricity \\
Heat & $\begin{array}{c}\text { isothermal heat } \\
\text { transfer }\end{array}$ & Dufour effect & Peltier effect & thermal conduction \\
& & & & \\
\hline
\end{tabular}

et al. has been unable to fit the experimental data of either of the two materials. Huyghe and Janssen [23] have developed a finite deformation quadriphasic theory which includes both electric current and electric potential, and which has turned out to fit experimental data of confined swelling and compression reasonably well, using material parameters consistent with other experimental data [24].

The key role of swelling in the behaviour of shales [17] and the associated problems of bore hole instability in shale drilling force petroleum engineers to develop continuum models of swelling shales. Numerous difficulties are associated with in situ measurement of relevant quantities around the borehole and with taking adequate samples of shale for subsequent testing in the laboratory. Therefore, there is a need for advanced models able to translate the experimental data available into predictions of the in situ situation. Unlike biological tissues, the shale is exposed to high overburden loads - in the order of hundreds of atmospheres - causing swelling forces other than osmosis to play a dominant role. Heidug and Wong [15] have developed a triphasic model which includes hydration forces in addition to osmosis, and have applied it to shales.

Table I gives an overview of direct and coupled flow phenomena induced by pressure, chemical potential, electrical potential and temperature gradients in charged porous media [16].

In this paper, we present a theory of swelling incompressible charged porous media. In order to simplify the mathematics as much as possible, a Lagrangian form of the entropy inequality [18] is used which leads to equations consistent with Biot's porous media theories in a more straightforward way than the more familiar Eulerian approach of Bowen [5]. The saturation and electroneutrality conditions are introduced by means of two Lagrange multipliers; the latter is physically interpreted as an electrical potential, the former as a pressure.

\section{Theory}

We assume all constituents $\alpha$ intrinsically incompressible, that is if one adds a mass $m$ of constituent $\alpha$ to the mixture, the added volume of the mixture is always proportional to the mass $m$, irrespective of the value $m$, of the initial composition of 
the mixture or of the state of deformation. The proportionality constant, the intrinsic density

$$
\rho_{i}^{\alpha}=\frac{\rho^{\alpha}}{n^{\alpha}},
$$

is constant. $\rho^{\alpha}$ represents the apparent density of the $\alpha$ th constituent. $n^{\alpha}$ is the volume fraction of the $\alpha$ th constituent. We use the word incompressible in the sense used by Bowen [5]: deformation of the constituents does not result in changes of intrinsic densities, nor do changes in mixing ratios result in changes of intrinsic densities. Although it is more common to express solute quantities in molar or mass values than in volume values, we will use volume fractions throughout this paper, to reduce the number of symbols introduced. Because of incompressibility, the ratio between mass and volume is constant, and the two quantities are interchangeable in the equations. Excluding mass transfer between constituents, the mass balance of each constituent is then written as

$$
\frac{\partial n^{\alpha}}{\partial t}+\nabla \cdot\left(n^{\alpha} \mathbf{v}^{\alpha}\right)=0,
$$

in which $\mathbf{v}^{\alpha}$ is the velocity of constituent $\alpha$ and $\nabla$ the Eulerian gradient operator. As we assume saturation, we find

$$
\sum_{\alpha} n^{\alpha}=1 .
$$

We will agree that summation over $\alpha$ is taken over all constituents and summation over $\beta$ or $\gamma$ is taken over all constituents but the solid. Differentiation of Equation (3) with respect to $t$ and substitution of the mass balance equations (2) yields the mass balance of the mixture

$$
\boldsymbol{\nabla} \cdot \mathbf{v}^{\mathrm{s}}+\sum_{\beta} \boldsymbol{\nabla} \cdot\left[n^{\beta}\left(\mathbf{v}^{\beta}-\mathbf{v}^{\mathrm{s}}\right)\right]=0 .
$$

The deformation gradient tensor $\mathbf{F}$ maps an infinitesimal material line segment in the initial state of the solid onto the corresponding line segment in the current state. The relative volume change from the initial to the current state is the determinant of the deformation gradient tensor $J=\operatorname{det} \mathbf{F}$. If we introduce volume fractions

$$
N^{\alpha}=J n^{\alpha}
$$

per unit initial volume, we can rewrite the mass balance equation (2) as follows:

$$
\frac{\mathrm{D}^{\mathrm{s}} N^{\alpha}}{\mathrm{D} t}+J \boldsymbol{\nabla} \cdot\left[n^{\alpha}\left(\mathbf{v}^{\alpha}-\mathbf{v}^{\mathrm{s}}\right)\right]=0
$$

when using the identity

$$
\frac{\mathrm{D}^{\mathrm{s}}}{\mathrm{D} t} J=J \nabla \cdot \mathbf{v}^{\mathrm{s}} .
$$


In Equations (6) and (7), $\mathrm{D}^{\mathrm{s}} / \mathrm{D} t=\partial / \partial t+\mathbf{v}^{\mathrm{s}} \cdot \nabla$ is the time derivative for an observer moving with the solid. The electrostatic interactions between ions and the charged solid are accounted for by means of an electroneutrality condition. Disturbances of charge neutrality are corrected in such media in a time lapse in the order of the quotient of the dielectric constant over the electrical conductivity of the medium. The order of magnitude of this time constant is $1 \mathrm{~ns}$ [11]. As we are not concerned with phenomena faster than $1 \mu \mathrm{s}$, we assume electroneutrality to be valid at all times. Magnetic fields generated by the electric fluxes through the medium are considered negligible. The electroneutrality condition requires that

$$
C^{f c}+\sum_{\beta} z^{\beta} C^{\beta}=0
$$

in which $z^{\beta}$ are the valences, $C^{f c}$ the fixed charge density per unit initial mixture volume and $C^{\beta}$ the current molar concentration of mobile ions in the solvent per unit initial mixture volume

$$
C^{\beta}=\frac{N^{\beta}}{\bar{V}^{\beta}},
$$

in which $\bar{V}^{\beta}$ are the partial molar volumes of the solvent and solutes. Equation (8) is rewritten in a more convenient differentiated form, using the fixity of the fixed charges $\left(\mathrm{D}^{\mathrm{s}} C^{f c} / \mathrm{D} t=0\right)$ and Equation (9) [23]

$$
\sum_{\beta} \frac{z^{\beta}}{\bar{V}^{\beta}} \nabla \cdot\left[n^{\beta}\left(\mathbf{v}^{\beta}-\mathbf{v}^{\mathrm{s}}\right)\right]=0 .
$$

Neglecting inertia, the momentum balance takes the form

$$
\boldsymbol{\nabla} \cdot\left(\boldsymbol{\sigma}^{\alpha}\right)^{C}+\boldsymbol{\pi}^{\alpha}=n^{\alpha} \mathbf{b}^{\alpha},
$$

which, after summation over all constituents, yields

$$
\boldsymbol{\nabla} \cdot(\boldsymbol{\sigma})^{C}=\sum_{\alpha} \boldsymbol{\nabla} \cdot\left(\boldsymbol{\sigma}^{\alpha}\right)^{C}=\mathbf{b},
$$

if use is made of the balance condition:

$$
\sum_{\alpha} \boldsymbol{\pi}^{\alpha}=\mathbf{0}
$$

$\boldsymbol{\sigma}^{\alpha}$ is the partial Cauchy stress tensor of constituent $\alpha, \boldsymbol{\pi}^{\alpha}$ the momentum interaction with constituents other than $\alpha, \mathbf{b}^{\alpha}$ the body force of constituent $\alpha$ per unit volume and $\mathbf{b}=\sum_{\alpha} n^{\alpha} \mathbf{b}^{\alpha}$ the body force of the mixture per unit volume. The partial Cauchy stress is defined for all constituents in the same way in accordance with standard literature, for example [12]. One should expect, for example, that electrostatic interactions cause significant forces between mobile ions. Balance of 
moment of momentum requires that the stress tensor $\boldsymbol{\sigma}$ be symmetric. If no moment of momentum interaction between components occurs, the partial stresses $\boldsymbol{\sigma}^{\alpha}$ are also symmetric. In this paper we assume all partial stresses to be symmetric. The balance of energy for the $\alpha$ th constituent reads

$$
n^{\alpha} \frac{\mathrm{D}^{\alpha} \epsilon^{\alpha}}{\mathrm{D} t}=\boldsymbol{\sigma}^{\alpha}: \nabla \mathbf{v}^{\alpha}-\nabla \cdot \mathbf{q}^{\alpha}+n^{\alpha} r^{\alpha}+\hat{\epsilon}^{\alpha},
$$

in which $\epsilon^{\alpha}$ is the partial internal energy of the $\alpha$ th constituent per unit volume, $\mathbf{q}^{\alpha}$ the heat flux of constituent $\alpha, r^{\alpha}$ the partial heat supply per unit volume constituent and $\hat{\epsilon}^{\alpha}$ the energy interaction with other constituents per unit volume. Total energy balance requires

$$
\sum_{\alpha}\left(\hat{\epsilon}^{\alpha}+\mathbf{v}^{\alpha} \cdot \pi^{\alpha}\right)=0
$$

Substitution of Equation (14) into Equation (15) yields an equivalent form of the total energy balance

$$
\frac{\mathrm{D}^{\mathrm{s}} \epsilon}{\mathrm{D} t}=\sum_{\alpha}\left\{\boldsymbol{\sigma}^{\alpha}: \nabla \mathbf{v}^{\alpha}-\nabla \cdot\left[\mathbf{q}^{\alpha}+n^{\alpha} \epsilon^{\alpha}\left(\mathbf{v}^{\alpha}-\mathbf{v}^{\mathrm{s}}\right)\right]-\mathbf{v}^{\alpha} \cdot \boldsymbol{\pi}^{\alpha}\right\}-\epsilon \boldsymbol{\nabla} \cdot \mathbf{v}^{\mathrm{s}}+r,
$$

in which

$$
\epsilon=\sum_{\alpha} n^{\alpha} \epsilon^{\alpha}
$$

is the internal energy of the mixture per unit volume and

$$
r=\sum_{\alpha} n^{\alpha} r^{\alpha}
$$

is the total heat supply of the mixture per unit volume. Under incompressible conditions, the entropy inequality for a unit volume of mixture reads [5]

$$
\frac{\mathrm{D}^{\mathrm{s}} \eta}{\mathrm{D} t}-\eta \nabla \cdot \mathbf{v}^{\mathrm{s}}+\sum_{\alpha}\left[\nabla \cdot\left(\frac{\mathbf{q}^{\alpha}}{\theta^{\alpha}}+n^{\alpha} \eta^{\alpha}\left(\mathbf{v}^{\alpha}-\mathbf{v}^{s}\right)\right)-\frac{n^{\alpha} r^{\alpha}}{\theta^{\alpha}}\right] \geqslant 0,
$$

in which $\eta^{\alpha}$ is the entropy of constituent $\alpha$ per unit volume constituent, $\eta=\sum_{\alpha} n^{\alpha} \eta^{\alpha}$ is the entropy of the mixture per unit mixture volume and $\theta^{\alpha}$ the temperature of the constituent $\alpha$. If the temperature field is assumed to be the same for all constituents, we find

$$
\frac{\mathrm{D}^{\mathrm{s}} \eta}{\mathrm{D} t}-\eta \nabla \cdot \mathbf{v}^{\mathrm{s}}+\boldsymbol{\nabla} \cdot\left[\frac{1}{\theta} \sum_{\alpha}\left(\mathbf{q}^{\alpha}+n^{\alpha} \eta^{\alpha} \theta\left(\mathbf{v}^{\alpha}-\mathbf{v}^{\mathrm{s}}\right)\right)\right]-\frac{r}{\theta} \geqslant 0,
$$


in which $\theta$ is the temperature of the mixture. Substitution of the energy balance (16) into the entropy inequality eliminates the heat supply $r$ from the inequality

$$
-\eta \frac{\mathrm{D}^{\mathrm{s}} \theta}{\mathrm{D} t}-\frac{\mathbf{q} \cdot \boldsymbol{\nabla} \theta}{\theta}+\sum_{\alpha}\left(-n^{\alpha} \frac{\mathrm{D}^{\alpha} \Psi^{\alpha}}{\mathrm{D} t}+\boldsymbol{\sigma}^{\alpha}: \boldsymbol{\nabla} \cdot \mathbf{v}^{\alpha}-\boldsymbol{\pi}^{\alpha} \cdot \mathbf{v}^{\alpha}\right) \geqslant 0,
$$

in which

$$
\Psi^{\alpha}=\epsilon^{\alpha}-\theta \eta^{\alpha}
$$

is the Helmholtz free energy of constituent $\alpha$ per unit volume constituent and

$$
\mathbf{q}=\sum_{\alpha}\left[\mathbf{q}^{\alpha}+n^{\alpha} \eta^{\alpha} \theta\left(\mathbf{v}^{\alpha}-\mathbf{v}^{\mathrm{s}}\right)\right]
$$

is an energy flux vector of the mixture. We introduce the strain energy function

$$
W=J \sum_{\alpha} n^{\alpha} \Psi^{\alpha}=J \sum_{\alpha} \psi^{\alpha}
$$

as the Helmholtz free energy of a mixture volume which in the initial state of the solid equals unity. $\psi^{\alpha}$ is the Helmholz free energy of constituent $\alpha$ per unit mixture volume. Rewriting the inequality (21) for the entropy production per initial mixture volume - that is, we multiply inequality (21) by the relative volume change $J$ - we find

$$
\begin{aligned}
& -J \eta \frac{\mathrm{D}^{\mathrm{s}} \theta}{\mathrm{D} t}-J \frac{\mathbf{q} \cdot \boldsymbol{\nabla} \theta}{\theta}-\frac{\mathrm{D}^{\mathrm{s}}}{\mathrm{D} t} W+J \boldsymbol{\sigma}: \boldsymbol{\nabla} \mathbf{v}^{\mathrm{s}}-J \boldsymbol{\nabla} \cdot \sum_{\beta}\left(\mathbf{v}^{\beta}-\mathbf{v}^{\mathrm{s}}\right) \psi^{\beta}+ \\
& \quad+J \sum_{\beta}\left[\nabla\left(\mathbf{v}^{\beta}-\mathbf{v}^{\mathrm{s}}\right): \boldsymbol{\sigma}^{\beta}-\left(\mathbf{v}^{\beta}-\mathbf{v}^{\mathrm{s}}\right) \cdot \boldsymbol{\pi}^{\beta}\right] \geqslant 0 .
\end{aligned}
$$

The entropy inequality should hold for an arbitrary state of the mixture, complying with the balance laws, incompressibility, saturation and electroneutrality. There are two ways to comply with these restrictions. One is substitution of the restriction into the inequality, resulting in the elimination of a field variable. The other is by introduction of a Lagrange multiplier. The balance laws and the incompressibility conditions (1) are accounted for by means of substitution. The differentiated forms of the saturation condition (4) and the electroneutrality (10) are accounted for by means of a Lagrange multiplier. The inequality (25) shows that the apparent densities $\rho^{\alpha}$, the body forces $\mathbf{b}^{\alpha}$ and the heat supplies $r^{\alpha}$ are already eliminated from the inequality. In other words the conditions of incompressibility (1) are fulfilled by choosing $\rho^{\alpha}=\rho_{i}^{\alpha} n^{\alpha}$, the momentum balances (11) are fulfilled by choosing $\mathbf{b}^{\alpha}$ accordingly and the energy balance is fulfilled by choosing the heat supply $r$ accordingly. Therefore, restrictions still to be fulfilled are the mass balances, saturation and the electroneutrality. The differentiated form of the saturation condition (4) is 
substituted by means of a Lagrange multiplier $p$

$$
\begin{aligned}
- & \eta_{0} \frac{\mathrm{D}^{\mathrm{s}} \theta}{\mathrm{D} t}-\frac{J \mathbf{v}^{q \mathrm{~s}} \cdot \nabla_{0} \theta}{\theta}-\frac{\mathrm{D}^{\mathrm{s}}}{\mathrm{D} t} W+J \boldsymbol{\sigma}^{\mathrm{eff}}: \boldsymbol{\nabla} \mathbf{v}^{\mathrm{s}}+ \\
& +J \sum_{\beta}\left[\boldsymbol{\sigma}^{\beta}+\left(p n^{\beta}-\psi^{\beta}\right) \mathbf{I}\right]: \boldsymbol{\nabla}\left(\mathbf{v}^{\beta}-\mathbf{v}^{\mathrm{s}}\right)+ \\
& +J \sum_{\beta}\left(\mathbf{v}^{\beta}-\mathbf{v}^{\mathrm{s}}\right) \cdot\left(-\boldsymbol{\nabla} \psi^{\beta}+p \boldsymbol{\nabla} n^{\beta}-\boldsymbol{\pi}^{\beta}\right) \geqslant 0,
\end{aligned}
$$

in which the effective stress $\boldsymbol{\sigma}^{\text {eff }}$ is defined as

$$
\boldsymbol{\sigma}^{\text {eff }}=\boldsymbol{\sigma}+p \mathbf{I},
$$

the heat flux $\mathbf{v}^{q s}$ through an area equal to unity in the initial state of the solid as

$$
\mathbf{v}^{q s}=\mathbf{F}^{-1} \cdot \mathbf{q}
$$

and the entropy per unit initial volume as

$$
\eta_{0}=J \eta
$$

Introducing the restriction (10) into inequality (26) by means of a Lagrange multiplier $\lambda$, yields

$$
\begin{aligned}
& -\eta_{0} \frac{\mathrm{D}^{\mathrm{s}} \theta}{\mathrm{D} t}-\frac{J \mathbf{v}^{q \mathrm{~s}} \cdot \nabla_{0} \theta}{\theta}-\frac{\mathrm{D}^{\mathrm{s}}}{\mathrm{D} t} W+J \boldsymbol{\sigma}^{\mathrm{eff}}: \boldsymbol{\nabla} \mathbf{v}^{\mathrm{s}}+ \\
& +J \sum_{\beta}\left[\boldsymbol{\sigma}^{\beta}+\left(\left(p+\frac{z^{\beta} \lambda}{\bar{V}^{\beta}}\right) n^{\beta}-\psi^{\beta}\right) \mathbf{I}\right]: \nabla\left(\mathbf{v}^{\beta}-\mathbf{v}^{\mathrm{s}}\right)+ \\
& \quad+J \sum_{\beta}\left(\mathbf{v}^{\beta}-\mathbf{v}^{\mathrm{s}}\right) \cdot\left[-\nabla \psi^{\beta}+\left(p+\frac{z^{\beta} \lambda}{\bar{V}^{\beta}}\right) \nabla n^{\beta}-\boldsymbol{\pi}^{\beta}\right] \geqslant 0,
\end{aligned}
$$

in which $\nabla_{0}=\mathbf{F}^{C} \cdot \nabla$ is the gradient operator with respect to the initial configuration. We choose as independent variables the Green strain $\mathbf{E}$, the temperature $\theta$, the Lagrangian form of the volume fractions of the fluid and the ions $N^{\beta}$, and of the relative velocities $\mathbf{v}^{\beta \mathrm{s}}=\mathbf{F}^{-1} \cdot\left(\mathbf{v}^{\beta}-\mathbf{v}^{\mathrm{s}}\right)$ and $\mathbf{v}^{q \mathrm{~s}}$. The dependent variables are the Helmholtz free energy per unit initial mixture volume $W$, the entropy per unit initial mixture volume $\eta_{0}$, the effective stress $\boldsymbol{\sigma}^{\text {eff }}$ of the mixture, the Helmholtz free energy $\psi^{\gamma}$ of constituent $\gamma$, the effective partial stresses $\boldsymbol{\sigma}^{\gamma}+\left(p+z^{\gamma} \lambda / \bar{V}^{\gamma}\right) n^{\gamma} \mathbf{I}$, the effective momentum interactions $\boldsymbol{\pi}^{\gamma}-\left(p+z^{\gamma} \lambda / \bar{V}^{\gamma}\right) \nabla n^{\gamma}$ and the temperature gradient $\boldsymbol{\nabla}_{0} \theta$. We apply the principle of equipresence, that is, all dependent variables depend on all independent variables, unless the entropy inequality requires otherwise:

$$
\begin{aligned}
& W=W\left(\mathbf{E}, \theta, N^{\beta}, \mathbf{v}^{\beta \mathrm{s}}, \mathbf{v}^{q \mathrm{~s}}\right), \\
& \eta_{0}=\eta_{0}\left(\mathbf{E}, \theta, N^{\beta}, \mathbf{v}^{\beta \mathrm{s}}, \mathbf{v}^{q \mathrm{~s}}\right),
\end{aligned}
$$




$$
\begin{aligned}
& \boldsymbol{\sigma}^{\mathrm{eff}}=\mathbf{F} \cdot \mathbf{S}^{\mathrm{eff}}\left(\mathbf{E}, \theta, N^{\beta}, \mathbf{v}^{\beta \mathrm{s}}, \mathbf{v}^{q \mathrm{~s}}\right) \cdot \mathbf{F}^{C}, \\
& \psi^{\gamma}=\psi^{\gamma}\left(\mathbf{E}, \theta, N^{\beta}, \mathbf{v}^{\beta \mathrm{s}}, \mathbf{v}^{q \mathrm{~s}}\right), \\
& \boldsymbol{\sigma}^{\gamma}+\left(p+\frac{z^{\gamma} \lambda}{\bar{V}^{\gamma}}\right) n^{\gamma} \mathbf{I}=\mathbf{F} \cdot \mathbf{S}^{\gamma}\left(\mathbf{E}, \theta, N^{\beta}, \mathbf{v}^{\beta \mathrm{s}}, \mathbf{v}^{q \mathrm{~s}}\right) \cdot \mathbf{F}^{C}, \\
& \boldsymbol{\pi}^{\gamma}-\left(p+\frac{z^{\gamma} \lambda}{\bar{V}^{\gamma}}\right) \nabla n^{\gamma}=\mathbf{F} \cdot \mathbf{p}^{\gamma}\left(\mathbf{E}, \theta, N^{\beta}, \mathbf{v}^{\beta \mathrm{s}}, \mathbf{v}^{q \mathrm{~s}}\right), \\
& \boldsymbol{\nabla}_{0} \theta=\mathbf{g}_{0}\left(\mathbf{E}, \theta, N^{\beta}, \mathbf{v}^{\beta \mathrm{s}}, \mathbf{v}^{q \mathrm{~s}}\right) .
\end{aligned}
$$

We apply the chain rule for time differentiation of $W$, which implies:

$$
\begin{aligned}
& -\left(\eta_{0}+\frac{\partial W}{\partial \theta}\right) \frac{\mathrm{D}^{\mathrm{s}} \theta}{\mathrm{D} t}-\frac{\mathbf{v}^{q \mathrm{~s}} \cdot \nabla_{0} \theta}{\theta}\left(J \boldsymbol{\sigma}^{\mathrm{eff}}-\mathbf{F} \cdot \frac{\partial W}{\partial \mathbf{E}} \cdot \mathbf{F}^{C}\right): \nabla \mathbf{v}^{\mathrm{s}}- \\
& -\frac{\partial W}{\partial \mathbf{v}^{\mathrm{s}}} \cdot \frac{\mathrm{D}^{\mathrm{s}}}{\mathrm{D} t} \mathbf{v}^{q \mathrm{~s}}+\sum_{\beta}\left\{-\frac{\partial W}{\partial \mathbf{v}^{\beta \mathrm{s}}} \cdot \frac{\mathrm{D}^{\mathrm{s}}}{\mathrm{D} t} \mathbf{v}^{\beta \mathrm{s}}+\right. \\
& +J\left[\boldsymbol{\sigma}^{\beta}+\left(\mu^{\beta} n^{\beta}-\psi^{\beta}\right) \mathbf{I}\right]: \nabla\left(\mathbf{v}^{\beta}-\mathbf{v}^{\mathrm{s}}\right)+ \\
& \left.\quad+J\left(\mathbf{v}^{\beta}-\mathbf{v}^{\mathrm{s}}\right) \cdot\left(-\boldsymbol{\nabla} \psi^{\beta}+\mu^{\beta} \nabla n^{\beta}-\boldsymbol{\pi}^{\beta}\right)\right\} \geqslant 0,
\end{aligned}
$$

in which $\mu^{\beta}$ are the electrochemical potentials of constituents other than the porous solid

$$
\mu^{\beta}=\frac{\partial W}{\partial N^{\beta}}+p+\frac{z^{\beta} \lambda}{\bar{V}^{\beta}} .
$$

Comparison of the above equation to the classical equations of electrochemistry indicates that the Lagrange multiplier $p$ can be interpreted as the fluid pressure and $\lambda$ as the electrical potential of the medium multiplied by the constant of Faraday. Inequation (38) should be true for any value of the state variables. In view of the set of independent variables, the first term of (38) is linear in the time derivative of the temperature $\mathrm{D}^{\mathrm{s}} \theta / \mathrm{D} t$, the third term is linear in the solid velocity gradient $\nabla \mathbf{v}^{\mathrm{s}}$, the fourth term linear in $\left(\mathrm{D}^{\mathrm{s}} / \mathrm{D} t\right) \mathbf{v}^{q \mathrm{~s}}$, the fifth term linear in $\left(\mathrm{D}^{\mathrm{s}} / \mathrm{D} t\right) \mathbf{v}^{\beta \mathrm{s}}$ and the sixth term linear in the relative velocity gradients $\nabla\left(\mathbf{v}^{\beta}-\mathbf{v}^{\mathrm{s}}\right)$. Therefore, by a standard argument [3], we find

$$
\begin{aligned}
& \eta_{0}=-\frac{\partial W}{\partial \theta}, \\
& \boldsymbol{\sigma}^{\mathrm{eff}}=\frac{1}{J} \mathbf{F} \cdot \frac{\partial W}{\partial \mathbf{E}} \cdot \mathbf{F}^{C}, \\
& \frac{\partial W}{\partial \mathbf{v}^{q s}}=0, \\
& \frac{\partial W}{\partial \mathbf{v}^{\beta \mathrm{s}}}=0,
\end{aligned}
$$




$$
\boldsymbol{\sigma}^{\beta}=\left(\psi^{\beta}-\mu^{\beta} n^{\beta}\right) \mathbf{I},
$$

leaving as inequality

$$
-\frac{J \mathbf{v}^{q \mathrm{~s}} \cdot \nabla_{0} \theta}{\theta}+\sum_{\beta} J\left(\mathbf{v}^{\beta}-\mathbf{v}^{\mathrm{s}}\right) \cdot\left(-\nabla \psi^{\beta}+\mu^{\beta} \nabla n^{\beta}-\boldsymbol{\pi}^{\beta}\right) \geqslant 0 .
$$

Equation (41) indicates that the effective stress of the mixture can be derived from a strain energy function $W$ which represents the free energy of the mixture. Equation (43) shows that the strain energy function cannot depend on the relative velocities. Thus, the effective stress of a charged porous medium can be derived from a regular strain energy function, which physically has the same meaning as in single phase or biphasic media, but which can depend on both strain and solute concentrations in the medium. According to Equation (44), the partial stress of the fluid and the solutes are scalars. Transforming the relative velocities to their Lagrangian equivalents, we find, instead of (45),

$$
-\frac{\mathbf{v}^{q \mathrm{~s}} \cdot \nabla_{0} \theta}{\theta}+\sum_{\beta} \mathbf{v}^{\beta \mathrm{s}} \cdot\left[-\nabla_{0} \psi^{\beta}+\mu^{\beta} \nabla_{0} n^{\beta}-\mathbf{F}^{C} \cdot \boldsymbol{\pi}^{\beta}\right] \geqslant 0 .
$$

If we assume that the system is not too far from equilibrium, we can express the dissipation (46) associated with relative flow of fluid, solutes and heat as a quadratic function of the relative velocities

$$
\begin{aligned}
& -\boldsymbol{\nabla}_{0} \psi^{\beta}+\mu^{\beta} \boldsymbol{\nabla}_{0} n^{\beta}-\mathbf{F}^{C} \cdot \boldsymbol{\pi}^{\beta}=\sum_{\gamma} \mathbf{B}^{\beta \gamma} \cdot \mathbf{v}^{\gamma \mathrm{s}}+\mathbf{B}^{\beta q} \cdot \mathbf{v}^{q \mathrm{~s}}, \\
& -\frac{\boldsymbol{\nabla}_{0} \theta}{\theta}=\sum_{\gamma} \mathbf{B}^{q \gamma} \cdot \mathbf{v}^{\gamma \mathrm{s}}+\mathbf{B}^{q q} \cdot \mathbf{v}^{q \mathrm{~s}},
\end{aligned}
$$

in which

$$
\left[\begin{array}{ll}
\mathbf{B}^{\beta \gamma} & \mathbf{B}^{\beta q} \\
\mathbf{B}^{q \gamma} & \mathbf{B}^{q q}
\end{array}\right]
$$

is a positive definite matrix of frictional tensors. Substituting Equations (11) and (44) into Equation (47) yields Lagrangian forms of the classical equations of irreversible thermodynamics

$$
\begin{aligned}
& -n^{\beta} \boldsymbol{\nabla}_{0} \mu^{\beta}-\mathbf{F}^{C} \cdot n^{\beta} \mathbf{b}^{\beta}=\sum_{\gamma} \mathbf{B}^{\beta \gamma} \cdot \mathbf{v}^{\gamma \mathrm{s}}+\mathbf{B}^{\beta q} \cdot \mathbf{v}^{q \mathrm{~s}}, \\
& -\frac{\boldsymbol{\nabla}_{0} \theta}{\theta}=\sum_{\gamma} \mathbf{B}^{q \gamma} \cdot \mathbf{v}^{\gamma \mathrm{s}}+\mathbf{B}^{q q} \cdot \mathbf{v}^{q \mathrm{~s}} .
\end{aligned}
$$

The boundary conditions are given by a no-jump condition of the temperature, the electrochemical potential of the ions and the fluid across the boundary and the momentum balance of the boundary. 


\section{Discussion}

The analysis presented in this paper differs in a few aspects from related work in the literature. We demonstrate that the constitutive behaviour needed for analysis of the deformation of swelling material (Equations (39)-(41), (50) and (51)) is entirely contained in the strain energy function $W$ and the frictional matrix $\mathbf{B}^{\beta \gamma}$. This is a more compact description than the more classical Eulerian analysis requiring separate free energy functions to be evaluated for each constituent (e.g. [5, 7]) and might save a great deal of tedious work for experimentalists responsible for quantifying these constitutive functions. The definition of the function $W$ as the Helmholtz free energy of a mixture volume equal to unity in the initial state of the solid is consistent with the classical definition of a strain energy function of a single phase material or of a saturated porous medium by Biot [4]. It is a straightforward extension of the work of Wilmansky on Lagrangian porous media models [20].

We apply the method of Coleman and Noll [3] to derive constitutive relationships from the second law of thermodynamics. This method has been criticized [21] and shown to be more restrictive than Mueller's method [19]. However, the criticisms are not shared by all experts in the field $[19,20]$, and for our purposes the use of either method is satisfactory.

Another feature distinguishes this thermodynamic analysis of swelling materials from the pioneering work of Lai et al. [7]. Lai et al. neglect to introduce the electroneutrality condition as a restriction on the second law of thermodynamics, although this condition mutually links the ion velocity gradients according to Equation (10). The electrical potential, obtained in our analysis as the Lagrange multiplier associated with the electroneutrality condition, is introduced in their analysis as an external volume force, which it is not. Our Lagrange multiplier $\lambda$ is an internally generated electric field adding up to external electric fields contained in the body force vector $\mathbf{b}^{\alpha}$.

Equations (39)-(41) are rewritten in their differentiated form

$$
\left[\begin{array}{c}
\mathrm{d} \boldsymbol{\sigma}^{\text {eff }} \\
\mathrm{d}\left(\mu^{\beta}-p-\frac{z^{\beta} \lambda}{\bar{V}^{\beta}}\right) \\
-\mathrm{d} \eta_{0}
\end{array}\right]=\left[\begin{array}{ccc}
\frac{\partial^{2} W}{\partial \mathbf{E}^{2}} & \frac{\partial^{2} W}{\partial N^{\gamma} \partial \mathbf{E}} & \frac{\partial^{2} W}{\partial \theta \partial \mathbf{E}} \\
\frac{\partial^{2} W}{\partial \mathbf{E} \partial N^{\beta}} & \frac{\partial^{2} W}{\partial N^{\gamma} \partial N^{\beta}} & \frac{\partial^{2} W}{\partial \theta \partial N^{\beta}} \\
\frac{\partial^{2} W}{\partial \mathbf{E} \partial \theta} & \frac{\partial^{2} W}{\partial N^{\gamma} \partial \theta} & \frac{\partial^{2} W}{\partial \theta^{2}}
\end{array}\right]\left[\begin{array}{c}
\mathrm{d} \mathbf{E} \\
\mathrm{d} N^{\gamma} \\
\mathrm{d} \theta
\end{array}\right]
$$

or

$$
\mathrm{d} \underline{\boldsymbol{y}}=\underline{\mathbf{C}} \mathrm{d} \underline{\boldsymbol{x}} .
$$

Assuming smoothness of the function $W$, the matrix $\underline{\mathbf{C}}$ should be symmetric. The existence of the function $W$ and the symmetry of $\underline{\mathbf{C}}$ is closely associated with the principle of reversibility. This principle means that if the changes occur at an infinitely slow rate, the work done to bring the material from the initial condition to its final state 
of strain and composition is independent of the path by which the final state is reached. In the absence of such a condition, an infinite amount of energy could be drawn from the material by loading and unloading it along a closed cycle. The above derivation generalises the approach of Huyghe and Janssen [23] to nonisothermal conditions in which temperatures are the same for all components. From the above equations several well-known physical theories can be derived [23], such as the Nernst equation for diffusion potentials, streaming potentials or the finite strain poroelasticity theory of Biot [4]. A field of application where temperature gradients may play an important role in swelling materials is for example human skin.

The reasons for choosing the quadriphasic theory [23] as a basis for the development are both experimental and conceptual. Alternative theories are available in the literature $[7,8,10,22]$. Sherwood and Heidug have developed, triphasic theories in which fixed charges are absent altogether and, hence, in which electrical effects are not present. Although this is a valid approach for specific applications, it is not sufficient for our purposes, involving materials with high fixed charge densities. Snijders et al. [10] have handled somewhat less stringent assumptions. Lai et al. [7] have developed a triphasic theory in which fixed charges are included. Although at the outset the theory is general when applying it to articular cartilage, they assume that deformations are infinitesimal. Similar assumptions are handled by [22]. Gu et $a l$. [9] have used the theory of Lai et al. in the context of one-dimensional permeation experiments. Those three papers use constitutive assumptions which lead to asymmetrical forms of the matrix $\mathbf{C}^{\beta \gamma}$. The chemical expansion stress $\mathbf{T}_{\mathrm{c}}$ as introduced by Lai et al. [7], for example, contributes to $\mathbf{C}^{\mathrm{s} \beta}$, while corresponding lower-diagonal terms $\mathbf{C}^{\beta \mathrm{s}}$ vanish. Although such an assumption is acceptable for infinitesimal theory, caution should be taken in extending such constitutive modelling to the finite strain domain. Moreover, recent comparison of experimental and numerical data on transient swelling [24] has not shown evidence of the existence of a chemical expansion stress.

\section{Acknowledgements}

The research of Dr J. M. Huyghe has been made possible through a fellowship of the Royal Netherlands Academy of Arts and Sciences. Part of this research was supported by the Technology Foundation (STW). The authors thank M. M. Molenaar for his valuable comments to this manuscript.

\section{References}

1. Biot, M. A.: General theory of three-dimensional consolidation, J. Appl. Phys. 12 (1941), 155-164.

2. Staverman, A. J.: Non-equilibrium thermodynamics of membrane processes, Trans. Faraday Soc. 48 (1952), 176-185.

3. Coleman, B. D. and Noll, W.: The thermodynamics of elastic materials with heat conduction and viscosity, Arch. Rational Mech. Anal. 13 (1963), 167-178. 
4. Biot, M.: Theory of finite deformation of porous solids, Indiana University Math. J. 21 (1972), 597-620.

5. Bowen, R. M.: Incompressible porous media models by use of the theory of mixtures, Int. J. Engng. Sci. 18 (1980), 1129-1148.

6. Richards, E. G.: An Introduction to Physical Properties of Large Molecules in Solution, Cambridge University Press, Cambridge, 1980.

7. Lai, W. M., Hou, J. S. and Mow, V. C.: A triphasic theory for the swelling and deformation behaviours of articular cartilage, J. Biomech. Engng. 113 (1991), 245-258.

8. Sherwood, J. D.: Biot poroelasticity of a chemically active shale, Proc. Roy. Soc. London A 440 (1993), 367-377.

9. Gu, W. Y., Lai, W. M. and Mow, V. C.: Transport of fluid and ions through a porous-permeable charged-hydrated tissue, and streaming potential data on normal bovine articular cartilage, J. Biomech. 26 (1993), 709-723.

10. Snijders, H., Huyghe, J. M. and Janssen, J. D.: Triphasic finite element model for swelling porous media, Int. J. Num. Meth. Fluids 20 (1995), 1039-1046.

11. Grodzinsky, A. J., Roth, V., Meyers, E., Grossman, W. D. and Mow, V. C.: The significance of electromechanical and osmotic forces in the non-equilibrium swelling behaviour of articular cartilage in tension, J. Biomech. Engng. 103 (1981), 221-231.

12. Bluhm, J. and de Boer R.: Effective stresses - a clarification, Arch. Appl. Mech. 66 (1996), 479-492.

13. Drost, M. R., Willems P., Snijders, H., Huyghe, J. M., Janssen, J. D. and Huson, A.: Confined compression of canine annulus fibrosus under chemical and mechanical loading, J. Biomech. Engng. 117 (1995), 390-396.

14. Oomens, C. W. J., Heus, H. de, Huyghe, J. M., Nelissen, L. and Janssen, J. D.: Validation of the triphasic mixture theory for a mimic of intervertebral disk tissue, Biomimetics 3 (1994), 171-185.

15. Heidug, W. K. and Wong, S.-W.: Hydration swelling of water absorbing rocks: a constitutive model, Int. J. Num. Anal. Meth. Geomech. 20 (1996), 403-430.

16. Yeung, A. T. and Mitchell, J. K.: Coupled fluid, electrical and chemical flows in soil, Geotechnique 43 (1993), 121.

17. Oort, E. van, Hale, A. H., Mody, F. K. and Sanjit, R.: Critical parameters in modelling the chemical aspects of borehole stability in shales and in designing improved water-based shale drilling fluids, Society of Petroleum Engineers, paper 28309, presented at the SPE 69th Annual Technical Conference and Exhibition, New Orleans, La., September, 1994.

18. Vankan, W. J., Huyghe, J. M., Janssen, J. D. and Huson, A.: Poroelasticity of saturated solids with an application to blood perfusion, Int. J. Engng. Sci. 34 (1996), 1019-1031.

19. Mueller, I.: Thermodynamics, Pitman, Boston, 1985.

20. Wilmansky, K.: Lagrangian model of two-phase porous material, J. Non-Equil. Thermodyn. 20 (1995), 50-77.

21. Woods, L. C.: Thermodynamic inequalities in continuum mechanics, IMA J. Appl. Math. 29 (1982), 221-246.

22. Simon, B. R., Liable, J. P., Pflaster, D., Yuan, Y. and Krag, M. H.: A poroelastic finite element formulation including transport and swelling in soft tissue structures, J. Biomech. Engng. 118 (1996), 1-9.

23. Huyghe, J. M. and Janssen, J. D.: Quadriphasic theory of swelling incompressible porous media, Int. J. Engng. Sci. 35 (1997), 793-802.

24. Frijns, A. J. H., Huyghe, J. M. and Janssen, J. D.: A validation of the quadriphasic mixture theory for intervertebral disc tissue, Int. J. Engng. Sci. 35 (1997), 1419-1429. 